\title{
Campylobacter jejuni-Bakteriämie bei einem Patienten mit Non-Hodgkin-Lymphom; Fallbericht und Literaturübersicht
}

\author{
Campylobacter jejuni-Septicaemia in a Patient with Non-Hodgkin-Lymphoma: \\ Case Report and Review of the Literature
}

\author{
Anke Oltmann', Rosemarie Blatz ${ }^{1}$, Ute Hegenbart², F.-B. Spencker ${ }^{1}$
}

Zusammenfassung: Vertreter der Gattung Campylobacter gehören weltweit zu den führenden EnteritisErregern. Extraintestinale Infektionen durch Campylobacter und verwandte Genera werden relativ selten beobachtet und betreffen meist immunkompromittierte Patienten. Insbesondere bei diesen Patienten wächst das Spektrum der mit Campylobacter assoziierten Infektionen, die nahezu jedes Organsystem betreffen können. Im Gegensatz zur meist selbstlimitierenden Enteritis ist hier mit ernsten Prognosen zu rechnen. Des Weiteren spielt Campylobacter eine kausale Rolle bei der Entstehung des Guillain-Barré-Syndroms und anderen postinfektiösen Komplikationen. Da der anspruchsvolle Organismus in der Routinediagnostik leicht übersehen werden kann, gilt seine Inzidenz als wesentlich unterschätzt. Gleichzeitig zeichnen sich erste Resistenzprobleme ab, so daß wir mit einem Fallbericht und einer Literaturzusammenfassung zum diagnostischen Bewußtsein für diesen Erreger beitragen möchten.

Schlüsselwörter: Campylobacter; Bakteriämie; Immunschwäche; Enteritis; Guillain-Barré-Syndrom.

Summary: Campylobacters are among the most common causes of enteric infections worldwide. Extraintestinal infections due to Campylobacter and related genera are rarely reported and found mainly in immunocompromised hosts. Especially in these patients the spectrum of extraintestinal infections attributed to Campylobacter species is expanding and may affect almost any body site. In contrast to the usually selflimiting enteritis, the prognosis here can be serious. Furthermore Campylobacter is involved in the development of Guillain-Barré-syndrome and other postinfectious complications. Due to fastidious growth requirements the incidence of Campylobacter infections

\footnotetext{
'Inst. f. Med. Mikrobiologie und Infektionsepidemiologie, Universität Leipzig

${ }^{2}$ Med. Klinik II, Abt. f. Hămatologie/Onkologie, Klinikum der. Universität Leipzig

Korrespondenzadresse: Anke Oltmann. Universität Leipzig, Institut für Medizinische Mikrobiologie und Infektionsepidemiologie, Liebigstr. 24, 04103 Leipzig. Germany. Tel.: 0341-97-15 242, Fax: +49-341-97-15 249. E-mail: olta@medizin.yni-leipzig.de

Eingegangen: 5. April 2000/Angenommen: 13. Oktober 2000
}

is supposed to be substantially underreported in routine diagnostic. Since at the same time problems with antibiotic resistance are evolving, we want to contribute to diagnostic awareness about this organism with a case report and a summary of current literature.

Keywords: Campylobacter; bacteremia; immunodeficiency; enteritis; Guillain-Barré syndrome.

\section{Erreger}

Erreger der Gattung Campylobacter sind gramnegative, nicht sporenbildende Stäbchen mit kommaförmig oder spiralig gebogener Gestalt. Die Keime sind unioder bipolar begeißelt und strikt mikroaerophil. Die Gattung umfaßt mehrere Spezies und Subspezies, von denen $C$. jejuni der wichtigste humanpathogene Vertreter ist. Campylobacter wurde erstmals 1947 - damals noch als Vibrio- bei einem septischen Abort gefunden [2], nachdem er bereits seit 1909 als tierpathogen bekannt war [1]. Die 1963 eingeführte Gattungsbezeichnung trennte Campylobacter von den Vibrionen ab. Die weltweit mit über $90 \%$ am häufigsten isolierte Spezies ist Campylobacter jejuni, gefolgt von $C$. fetus und C. coli. Campylobacter jejuni und Campylobacter coli verursachen enteritische Krankheitsbilder, während Campylobacter fetus fast ausschließlich extraintestinal gefunden wird. Der Anteil seltenerer Spezies wie Campylobacier upsaliensis oder Campylobacter lari kann überregional sehr variieren $[3,8,27]$.

\section{Vorkommen und Infektion}

Erst die Möglichkeit der selektiven Isolation aus Stuhl 1972 offenbarte die weltweite Verbreitung von $\mathrm{Cam}$ pylobacter-Spezies als Enteritis-Erreger [3, 4]. An Häufigkeit übertrifft allein $C$. jejuni nach Daten aus den USA die Gesamtzahl an Enteritiden durch Salmonella spp., Shigella spp. und Escherichia coli 0157:H7 [5]. In Deutschland, Spanien und in der Schweiz ist $C$. jejuni der zweithäufigste Durchfallerreger $[6,7]$. Aufgrund der anspruchsvollen Diagnostik des Erregers, einerseits und einer hohen Dunkelziffer milder Verläufe ohne Diagnostik gelten die offiziellen Daten als weit unterschätzt. Angaben schwanken zwi- 
schen 5-6 und geschäitzten 1000 pro 100.000 Einwohner und Jahr [3.5.8]. Dic Meldeinzidenzraten liegen hicrzulande zwischen 10 und 50 pro 100.000 [9]. Eine Bevorzugung des männlichen Geschlechtes bei Campy/mbacter-Intektionen wird von mehrerế Autoren notiert $[3,8]$. Die höchste altersspezifische Infektionsrate weisen in Industrieländern Säuglinge unter einem Jahr und junge Erwachsene auf mit einem Erksankungsgipfel in der warmen Jahreszeit. In Entwicklungsländern sind vor allem Klcinkinder betroffen. Erregerreservoir ist weltweit die Darmschleimhaut von Nutz- und Haustieren, vor allem Geflügel, ebenso wie eine großc Vielfalt an Wildtieren bis hin zu Nagern und Reptilien [9, 11].

Die Übertragung erfolgt oral über unpasteurisierte Milch. kontaminiertes Wasser und vor allem ungenügend gegartes Fleisch bzw. Geflügel $(50-70 \%$ der Fälle) $[3,5,12,47]$. Hygiene im Umgang insbesondere mit Gefliigelfleisch, ausreichende Garzeiten, die Meidung unpasteurisierter Milch und nicht zuletzt Trinkwasserhygiene haben daher insbesondere für abwehrschwache Patienten und Kinder präventive $\mathrm{Be}$ deutung.

Die Infektionsdosis ist nicht genau definiert. $\mathrm{Ab}$ einer Keimzahl von $10^{3}$ ist mit Symptomen zu rechnen, die $\operatorname{ID}_{10}$ ist damit höher als bei Shigellen $[3,51]$. Die Inkubationszeit liegt bei 2-7 Tagen und nach einer Infektion mit ist mit einer Keimausscheidung für 2-4 Wochen zu rechnen (bei Immunkompromittierten länger). Infektionen sind meist sporadisch, eine fäkalorale Verbreitung von Mensch zu Mensch ist ungewöhnlich [5, 9]. Bei Ausbrüchen ist der Ursprung oft schwierig nachzuweisen. [13, 14, 15, 48].

Bakteriämie mit Campylobacter (auch hier führt $C$. jejuni) wird nur bei einem sehr geringen Teil der Infektionen nachgewiesen: in einer US-amerikanischen Studie [5] stammten 0,4\% aller Isolate von einer Bakteriämie, Skirrow fand eine Bakteriämie in $0,15 \%$ der intestinalen Campylobacter-Infektionen [5, 8]. Noch seltener ist der Nachweis anderer extraintestinaler Manifestationen, wobei auch hier von einer größeren Dunkelziffer ausgegangen werden kann.

\section{Pathogenität}

Campylobacter spp. verursachen eine entzündliche Enteritis. Als Virulenzfaktoren werden neben Motilität und bislang nur bei Primaten gesicherter Invasivität [5, $18,27,50]$, LPS, Inhibitoren der Phagozytenaktivität und noch ungeklärte Mechanismen vermutet [3,16]. Die Existenz eines relevanten Enterotoxins wird eher bezweifelt $[5,19,51]$. Man geht davon aus, daß eine intestinale Kolonisation Ausgangspunkt für Bakteriämie und septische Absiedelungen mit $C$. fetus und vermutlich auch $C$. jejuni ist $[3,7,47,51]$. Für die $\mathrm{Be}-$ grenzung einer Campylobacter-Infektion spielen unspezifische und spezifische Abwehrfaktoren eine Rolle wie Serumbakterizidie, Polymorphkernige und Makrophagen, Komplement und spezifische Antikörper [2,
5]. Im retikuloendothelialen System insbesondere der Leber wird Campylobacter phagozytiert, was ein gehäuftes Vorkommen von Bakteriämien bei Patienten mit Leberzirrhose oder auch nach Splenektomie erklärt $[47,49]$. C. jejuni gilt als empfindlich gegenüber bakteriziden Serumbestandteilen [2], wobei Unterschiede zwischen intestinalen und extraintestinalen Isolaten letztere mit höherer Serumresistenz - beschrieben wurden. Blaser $[10,50]$ legte dar, daß eine Bakteriämie bei Gesunden entweder transient im Rahmen einer Enteritis oder durch serumresistente $C$. jejıni-Stämmen bedingt ist. Bei Risikopatienten dagegen sind die meisten extraintestinalen. Isolate serumempfindlich und als Opportunisten anzusehen. $C$. fetus . ist grundsätzlich serumresistent auf Grund einer kapselähnlichen, oberflächlichen Proteinstruktur. die die C3-Bindung hemmt [47]. Dies korreliert mit einem relativ häufigeren Vorkommen von Bakteriämie und extraintestinalen Infektfoci $[3,8,51]$. Als ursächlich für anhaltende Bakteriämie wurde ein intrazelluläres Überleben der Keime in Makrophagen und Neutrophilen diskutiert [5]. Neuere Untersuchungen mit zytokinstimulierten Monozyten bestätigen dies nicht [16, 20], sondern zeigen ein in vitro mangelhaftes bakterizides Potential bei $10 \%$ der untersuchten menschlichen Makrophagenpopulation. Dieser individuell prädisponierende Faktor erweist sich als unabhängig von der Campylobacter-Spezies [20]. Die klinischen Daten mit prädisponierenden Faktoren auf Patientenseite wie HIV-Infektion, Leberzirrhose oder Hypogammaglobulinämie legen die Vermutung nahe, daß sowohl zelluläre als auch humorale Abwehrkräfte eine Rolle spielen. Die dem Alter abnehmende Schwere der Erkrankung in Entwicklungsländern mit hoher Durchseuchung weist auf die Möglichkeit einer spezifischen Immunität hin [5].

\section{Symptomatik}

Das Spektrum der Symptome einer Campylobacter jejuni-Enteritis reicht von geringfügiger Diarrhö oder abdominellen Schmerzen bis zu schweren, teilweise blutigen Diarrhöen mit Fieber, Erbrechen und abdominellen Krämpfen, eventuell begleitet von grippeähnlichen Symptomen. Schätzungsweise $25 \%$ aller Campylobacter-Enteritiden verlaufen inapparent. Die Krankheitsdauer beträgt bis zu einer Woche $[3,5,51]$. Da ein extraintestinaler Nachweis von Campylobacter insgesamt selten ist (s. o.), dominieren in der Literatur Fallberichte neben Übersichten global sehr heterogener Kohorten mit selten mehr als 30 Bakteriämien. Skirrow [8] überblickte 394 Campylobacter-Bakteriämien (darunter $89 \%$ C. jejuni/C. coli, $8.6 \%$ C. fetus) in einein Zehnjahreszeitraum in England, Guerrant [27] untersuchte 1978 in den USA 91 Fälle und Pigrau [7] 58 Fälle $(7 \%$. C. fetus) zwischen 1979 und 1996 in Spanien. Ein Überwiegen von $C$. fetus bei Guerrant im Gegẹnsatz zu späteren Untersuchungen gilt heute als Artefakt [8]. Zwischen transienter Bakteriämie und 
Sepsis wird in der Literatur selten unterschieden. Enteritissymptomatik besteht zeitgleich in etwa $70 \%$ der Fälle von Campvlobacter jejuni-Bakteriämie bei fäkalem Erregernachiveis in etwa 40\% [8]. Im Falle von $C$. fetus fehlt die Enteritis meist [8, 21]. Außer Bakteriämie ${ }^{-}$wurden intestinale Komplikationen wie Cholezystitis. Pankreatitis, akute Hepatitis oder spontane Peritonitis bei Leberzirrhose beschrieben. Extraintestinale Manifestationen einer Campylobacter jejuni-Infektion umfassen bislang Meningitis, purulente Arthritis, septische Aborte, Endokarditis, Salpingitis, Osteomyelitis, Hüft-und Gefäßprotheseninfektion und Zellulitis [3, 7, 8, 19, 23, 24, 25, 26, 27, 30]. Für Campylobacter fetus wird auf Grund von Thrombophlebiten und Vaskulitiden eine Endothelaffinität postuliert [8, 51. 53]. Pulmonale Affektionen wurden für beide Spezies plausibel beschrieben $[7,17,28,49]$. Meist läßt sich eine Begleiterkrankung eruieren. Aborte und Meningitis [21] sind jedoch für auch für zuvor gesunde Patienten dokumentiert und ein Hinweis auf begünstigte Absiedelungsorte [47].

\section{Prädisposition}

Von den Patienten mit extraintestinaler Campylobacter-Infektion weisen je nach Autor und Definition zwischen $28 \%$ und $93 \%$ (in der neueren Literatur meist um $60-80 \%$ ) immunschwächende Vorbedingungen auf [7. 8, 25, 29]. Häufige Prädispositionen sind: Hypogammaglobulinämie, Neoplasien, immunsuppressive Therapie, HIV-Erkrankung in späteren Stadien mit niedriger CD4-Zahl, Leberzirrhose und Säuglingsalter unter einem Jahr. Genannt werden auch Lupus erythematodes, Splenektomie bei Thalassämie, Transplantation, lange Hospitalisierung. Niereninsuffizienz, Gefäßerkrankungen oder Unterernährung $[3,7,21,17$, 30. $31,32,49]$. Hinzu kommen auf Reisen in Endemiegebiete aquirierte Infektionen. Beim Faktor höheres Lebensalter variiert die Altersgrenze je nach Autor, so daß eine allgemeine Aussage schwierig ist. Zur Etablierung einer intestinalen Kolonisierung als Ausgangspunkt für Infektionen kann auch eine reduzierte Magensäureproduktion - unter säureblockierender Therapie beitragen [28, 47]. Allgemein sind schwere Infektionsverläufe und extraintestinale Infektionen bei immumkompromittierten Patienten häufiger und es kommt zu rekurrierenden Verläufen. Diese Tendenzen legt Tee [17] in einer vergleichenden Studie von C. jejuni-Bakteriämie bei 9 HIV-Infizierten und 12 Nichtinfizierten dar. Schuster et al. [33] beschreiben dagegen eine komplett asymptomatische Bakteriämie durch $C$. jejuni bei einer Lymphompatientin vor Knochenmarkstransplantation: Der Anteil der C. fetus-Isolate ist bei immunkompromittierten Patienten relativ höher, überwiegend extraintestinalen Ursprungs und meist klinisch signifikant $[3.8,17,28,34,35]$. Die Inzidenz einer $C$. jejuni-Infektion wird bei AIDS-Kranken um den Faktor 40 gegenüber der Normalbevölkerung gesteigert angegeben, die der Bakteriämie-Inzidenz auf $5 \%$ bis $8 \%$ gegenüber max. $1,6 \%[17,36]$.

\section{Verlauf}

Eine Campylobacter-Enteritis bei Gesunden ist sclbstlimitierend. Die Letalität im Patientengut mit extraintestinalen Infektionen betrug bei Skirrow 2,5\%. Allos gibt 2.4 tödliche Verläufe pro $1000 \mathrm{C}$. jejuni-Infektionen an, Pigrau 10,5\% bei allerdings $93 \%$ Patienten mit Prädisposition [7, 24. 30]. Meyricux beschreibt einen septischen Schock mit Multiorganversagen durch $C$. jejuni nach Splenektomie bei Thallassämie [31]. Deutlich erhöht erscheint die Letalität in Verbindung mit Campvlobacter-Bakteriämie bei HIV-Patienten [17, $35,36]$ mit $33 \%$ bis $75 \%$.

Es mehren sich die Hinweise auf einen Zusammenhang zwischen $C$. jcjuni Infektion und dem GuillainBarré-Syndrom (GBS) [30. 15, 20]. Die Häufigkeit dieser Folgeerkrankung läßt sich ebenso schwer abschätzen wie die Gesamtzahl an Campylobacter-Infektionen. Eine kürzlich durchgemachte $C$. jejuni Infektion läßt sich serologisch bei immerhin 20-40\% der GBS-Patienten nachweisen. Ein überproportional hoher Anteils des Penner-Serotyps O:19 bei GBS in japanischen, deutschen und amerikanischen Studien erhärtet den Zusammenhang [37]. Des Weiteren wird eine reaktive Arthritis (meist bei HLA-B-27-positiven Personen) zu den postinfektiösen Komplikationen einer Campylobacter-Infektion gezählt [4]. Beschrieben wurden auch Hämolytische Anämie. Karditis und Enzephalopathie [3].

\section{Diagnostik}

Berichte über Campylobacter-Bakteriämie enthalten meist wenig Angaben zur genauen Methodik. In vielen Berichten gelang die Anzucht mit dem radiometrischen Bactec 460 System, nahezu ausschließlich in aeroben Flaschen. Die relative Anzuchtrate für Canpylobacter in gebräuchlichen Blutkultursystemen erscheint gut [39]. Experimentelle Vergleiche verschiedener Blutkultursysteme ergaben Unterschiede in der Detektionsdauer für verschiedene Campylobacter-Spezies. Carnahan [38] et al. isolierten im BacT/Alert System einen $C$. upsaliensis nach 4,8 Tagen. Andere Berichte gehen von durchschnittlich 3 bis zu 10 Tagen Detektionsdauer für $C$. jejuni aus $[39,40]$. Bei entsprechendem Verdacht lohnen sich Acridinfärbung, verlängerte Bebrütungsdauer und die Berücksichtigung mikroaerophiler Bedingungen bei terminalen Subkulturen. Bei anderen Materialien von entsprechenden Patienten sollten mikroaerophile Bedingungen in die diagnostischen Überlegungen einbezogen werden. $C$. jejıni wächst bevorzugt bei $42^{\circ} \mathrm{C}$. Mit der Filtrationsmethode und antibiotikafreien (cephalotinfreien) Ansatzmedien läßt sich die Detektion von Nicht-jejuni-Spezies aus dem Fäzes verbessern. Insbesondere $C$. fetus ist cephalotinempfindlich und wächst besser bei $37^{\circ} \mathrm{C}$. Blaser [47] vermutet, daß dieser Keim erheblich unterschätzt wird wegen der gebrüuchlichen, antibiotikahaltigen Medien und Inkubation bei $42^{\circ} \mathrm{C}$. Für epidemiologische Untersuchungen stehen Serotypisierung, 
Lysotypic und molekularbiologische Methoden zur Verfügung. Dic Untersuchung der ...Serumresistenz" ist hislang nicht einheitlich standardisiert. Ein Antikörpernachweis ist möglich $[28,43]$.

\section{Therapie}

Dic Bakteriäinie durch Campylobacter ist im Rahmen einer Enteritis oft als transient zu betrachten. Gerade bei immungeschwächten Patienten, schweren Verlaiufen oder Schwangcren ist cine Therapie jedoch angebracht aufgrund der oben aufgeführten Komplikationen. Imipenem, eventuell in Kombination mit Gentamicin, ist für die Therapie septischer und extraintestinaler Affektionen gut geeignet, alternativ stehen Chinolone. Tetrazykline, Ampicillin oder Erythromycin zur Wahl $[17,47]$. Bei Erregern aus Endemiegebieten wie Spanien. Thailand und Mexiko zeichnet sich eine zunehmend schwierige Resistenzlage ab, was auf den Einsatz von Antibiotika in der Tierzucht und im Einzelfall auf eine entsprechende Vorbehandlung des Patienten z. B. bei HIV zurückzuführen ist. So ist in Spanien für.C. jejuni mit $82 \%$. Cephalotinresistenz, $20 \%$ Ampicillinresistenz und bereits 54\% Chinolonresistenz zu rechnen $[5,34,44]$. Es wird eine multifaktorielle Resistenz mit Punktmutation im gyrA-Gen sowie veränderten Zellmembranproteinen angenommen. $C$. fetus ist im allgemeinen noch gut empfindlich, Chinolonresistenz kommt bei entsprechend vorbehandelten Patienten jedoch vor [34]. Bei HIV-Patienten wird bereits eine parenterale Therapie mit Imipenem oder einem Aminoglykosid in Kombination mit einer oralen, mehrwöchigen Therapie der Kolonisation z. B. mit Chinolonen empfohlen [28, 47]. Rifabutin-Prophylaxe gegen den Mycobacterium-avium-Komplex scheint bei HIV-Patienten auch einen günstigen Einfluß auf die Inzidenz von Campylobacter-Infektionen zu haben [52].

\section{Fallbericht}

Der 72jährige Schlosser wurde im März 1999 wegen eines Ulcus ventriculi mit histologischem Nachweis von Helicobacter pylori ambulant behandelt. Im Verlauf der internistischen Nachbetreuung wurde der Patient mit einer generalisierten Lymphknotenschwellung, zunehmendem Aszites und Ikterus auffällig. Bis auf zurückliegende Appendektomie, Schädelbasisbruch 1962 und eine benigne Prostatahyperplasie war der Patient bis dato gesund gewesen. Nach Voruntersuchungen in einem auswärtigen Krankenhaus wurde der Patient mit Verdacht auf ein Non-Hodgkin-Lymphom (NHL) an das Universitätsklinikum Leipzig überwiesen. Hier wurde mittels Beckenkammbiopsie die Markinfiltration eines NHL vom AILD (angioimmunoblastische Lymphadenopathie)-Typ gesichert mit ubiquitären Lymphknotenvergrößerungen und Splenomegalie, Pleuraergüssen sowie Aszites. Paraklinisch bestanden eine Anämie bei sekundärer Autoimmunhämolyse. Thrombozytopenie, Leukozytose von $12800 / \mu 1$ und eine Hypergammaglobulinämie. Ende April erhielt der Patient den ersten Zyklus einer Chemotherapie nach dem CHOP-21-Schema (Vincristin, Cyclophosphamid, Doxorubicin und Prednisolon) mit $75 \%$ der Dosis üher 5 Tage neben begleitender symptomatischer Therapie. Am letzten Tag der zytoreduktiven Therapie, die gut toleriert wurde, entwickelte der Patient Temperaluren bis $39^{\circ} \mathrm{C}$. Er erhielt Imipenem $3 x$ $0.5 \mathrm{~g}$ i.v und entfieberte innerhalb von Stunden. Im Fieberanstieg wurden ein . Paar Blutkulturen (acrob/anaerob. BacT/Alert, Organon Teknika) gewonnen. Die aerobe Flasche des Sets wurde innerhalb eines Tages mit typischer Wachstumslogarithmik und Reflektionseinheiten von $>1900$ als positiv angezeigt. Im Grampräparat wurde zunächst kein Erreger erkannt, mit Acridinorange- und verlängerter Fuchsinfärbung ließen sich jedoch spiralig gewundene Bakterien darstellen. Mikroaerophile Bedingungen waren bei der routinemäßigen Abimpfung der Blutkulturen nicht einbezogen worden. Von äußerst spärlichem aeroben und anaeroben Wachstum ließ sich der Erreger jedoch mikroaerophil fortzüchten und wurde mittels API Campy-System (BioMérieux, Nürtingen) als Campylobacter jejuni ssp. jejuni identifiziert. Im E-Test wurde er als sensibel für Roxithromycin und Imipenem und resistent gegen Ciprofloxacin getestet. Diarrhö bestand zu keinem Zeitpunkt. Begleitend untersuchten wir Serum des Patienten. Während die KBR für Campylobacter jejuni wegen Eigenhemmung nicht beurteilbar war, fielen Westernblots für IgG und IgA negativ aus. Dagegen sprach die Befundkonstellation bei Helicobacter pylori mit positivem IgM-und IgA-ELISA und grenzwertigem IgG für eine aktive Infektion. Der Patient wurde wenige Tage später zur ambulanten Fortsetzung der Chemotherapie bei subjektivem Wohlbefinden und klinisch regredientem Befund entlassen.

\section{Kommentar}

Wir stellten einen Patienten vor, bei dem es unter zytotoxisch-immunsuppressiver Therapie eines Lymphoms zu einer symptomatischen Bakteriämie mit Campylobacter jejuni kam. Als Ausgangspunkt kann nach vorliegenden Erkenntnissen eine intestinale Kolonisation (oder inapparente Infektion) mit $C$. jejuni gelten, die durch eine laufende Omeprazolgabe wegen Ulkuskrankheit noch begünstigt wurde. Die ..Serumresistenz" des Stammeș wurde nicht untersucht. Die Antikörperserologie war zur Diagnosestellung bei diesem Patienten nicht hilfreich, was bei einem immunkompromittierten Patienten nicht überrascht. Geht man von intestinaler Kolonisation als Sepsisherd aus, kann die Serologie von vornherein nur eine untergeordnete Rolle spielen. Das zur kalkulierten Intervention gewählte Imipenem ist für Campylobacter gut wirksam und war in diesem Fall zur Prävention septischer Komplikationen bei einem immunkompromittierten Patienten geeignet. Die Überlegung einer langfristigen, oralen Therapie mit Chinolonen analog den Empfehlúngen bei $C$. fetus und HIV (s. o.) wird durch ver- 
breitete Chinolonresistenz erschwert, die auch bei diesem C. jejuni-Stamm vorlag. Die Anzucht des Erregers gelang mit dem oben erwähnten System außerordentlich schnell. Eine ähnlich kurze Detektionszeit wie im vorliegenden Fall fanden wir in der Literatur nur in einem weiteren Fall [41]. wobei für viele andere Keime eine kürzere Detektionszeit im BacT/Alert System gegenüber anderen Systemen beschrieben wird [42]. Hervorzuheben ist, daß die Infektion ohne die Acridinorange-Färbung möglicherweise nicht erkannt worden wäre.

Eine extraintestinale Campylobacter-Infektion bleibt ein eher seltenes Ereignis, für das jedoch eine relativ hohe Dunkelziffer angenommen werden kann. Die beschriebenen Dispositionen sowie verschiedene Immundefekte lassen mit einer Zunahme der Prävalenz rechnen. Gleichzeitig werden neue, verwandte Genera und Spezies mit ähnlichem Erkrankungsspektrum beschrieben. Umso wichtiger ist es, die Entität der Campvlobacter-Infektionen im beschriebenen Patientengut zu bedenken, da abgesehen von Blutkulturen nur eine gezielte Diagnostik Erfolg verspricht und die klinische Signifikanz dieser Erreger aufklären kann.

\section{Literatur}

1. MacFadyean F, Stockman S. Report of the Department Committee appointed by the Board of Agriculture and Fisheries to inquire into epizootic abortion. His Majesty's Stationary Office, London 1909; Vol. 3.

2. Vinzent R, Dumas J, Picard N. Septicémia grave au cours de la grossesse due à un vibrion. Avortement consécutif. Bull Acad Nat Nied 1947:131:90.

3. Allos BM, Blaser MJ. Campylobacter jejuni and the expanding spectrum of related infections. Clin Infect Dis 1995:20:1092-101.

4. Bremell T, Bjelle A, Svedhem A. Rheumatic symptoms following an outbreak of Campylobacter enteritis: a five-year follow-up. Ann Rheum Dis 1991;50:934-8.

5. Blaser MJ. Epidemiologic and Clinical Features of Campylobacter jejuni Infections. J Infect Dis 1997;176 (Suppl 2):103-5.

6. Ruef C, Fäh L, Caduff F. Campylobacter jejuni: Sepsis und Meningitis bei einer Erwachsenen ohne Risikofaktoren. Schweiz Med Wochenschr 1988:118:302-3047.

7. Pigrau C, Bartolome R, Almirante B, Planes AM Gavalda J, Pahissa A. Bacteremia due to Gampylobacter species: clinical findings and antimicrobial susceptibility patterns. Clin Infect Dis 1997:25:1414-20.

8. Skirrow MB, Jones DM, Sutcliffe E, Benjamin J. Campylobacter bacteraemia in England and Wales, 1981-91. Epidemiol Infect 1993;110:567-73.

9. Ratgeber Infektionskrankheiten. Campylobacter-Infektionen. Epid Bull 1999:35:259-61.

10. Blaser MJ, Perez G, Smith PF, Patton C, Tenover FC, Lastovica AJ. Wang WIL. Extraintestinal Campylobacter jejuni and Campylobacter coli infections: host factors and strain characteristics. J Infect Dis 1986;153:552-9.

11. Smibert RM. Genus Camylobacter. In: Krieg NR, Holt HG, eds. Bergey's manual of systematic bacteriology, Vol. 1. Baltimore: Williams \& Wilkins, 1984:111-8.

12. Ono K, Yamamoto K. Contamination of meat with Campylobacter jejuni in Saitama, Japan. Int Food Microbiol 1999;47:211-9.

13. Shandera W, Tormey MP, Blaser MJ. An outbreak of bacteremic Campylobacter jejuni infection. Mount Sinaj $J$ Med 1992:59:53-6.

14. Outbreak of Escherichia coli $0157: \mathrm{H} 7$ and Campylobacter among attendees of the Washington County Fair-New York, 1999. Morb Mortal Wkly Rep 1999:48:803-5.
15. Merritt A, Miles R, Bates J. An outbreak of Campylobacter enteritis on an island resort. north Queensland. Commun Dis Intell 1999:23:215-9.

16. Pancorbo PL, Gallego AM, de Pablo M. Alvarez, C. Ortega E. de Cienfuegos GA. Inflammatory and phagocytic response to experimental Campylobacter jejuni 1 infection in mice. Microbiol Immunol 1994:38:89-95.

17. Tee W, Mijch A. Campylubacter jejuni bacteremia in Human Immunodeficiency Virus (HIV)-infected and non-infected patients: comparison of clinical features and review. Clin Infect Dis 1998:26:91-6.

18. Konkel ME, Kim BJ, Rivera-Amill V. Garvis SG. Bacterial secreted proteins are required for the internalization of Campylobacter jejuni into cultured mammalian cells. Mol Microbiol 1999:32:691-701.

19. Vermeij CG, van Dissel J, Veenendaal RA, Lamers CBHW, van Hoek B. Campylobacter jejuni peritonitis in a patient with liver cirrhosis. Eur J Gastruent Hepatol 1996:8:1219-21.

20. Wassenaar TM. Engelskirchen M, Park S, Lastovica A. Differential uptake and killing potential of Campylobacter jejuni by human peripheral monocytes/macrophages. Med Microbiol Immunol 1997;186:139-44.

21. Dhawan VK. Ulmer DD, Nachum R, Rao B. See RC. Campylobacter jejuni septicemia-epidemiology, clinical features and outcome. West J Med 1986;144:324-8.

22. Meyer A, Stallmach T, Goldenberger D. Altwegg M. Lethal maternal sepsis caused by Campylobacter jejuni: pathogen preserved in placenta and identified by molecular methods. Mod Pathol 1997:10:1253-6.

23. Peterson MC, Farr RW, Castiglia M. Prosthetic hip infection and bacteremia due to Campylobacter jejuni in a patient with AIDS. Clin Infect Dis 1993:16:439-40.

24. Ezpeleta C, Rojo de Ursua P, Obregon F, Goñi , Cisterna R. Acute pancreatitis associated with Campylobacter jejuni bacteremia. Clin Infect Dis 1992:15:1050.

25. Schonheyder H, Sogaard P. Fredriksen W. A survey of Campylobacter bacteremia in three Danish counties, 1989 to 1994. Scand J Infect Dis 1995;27:145-8.

26. Korman TM, Varley CC, Spelman DW. Acute hepatitis associated with Campylobacter jejuni bacteraemia. Eur J Clin Microbiol Infect Dis 1997; 17:678-9.

27. Guerrant RL. Lahita RG, Winn WC. Roberts RB. Campylobacteriosis in man: Pathogenic mechanisms and review of 91 bloodstream infections. Am J Med 1978:65:584-92.

28. Ichiyama S, Hirai S, Minami T, Nishiyama $Y$, Shimizu S, Shimokata K, Ohta M. Campylobacter fetus subspecies fetus cellulitis associated with bacteremia in debilitated hosts. Clin Infect Dis 1998;27:252-5.

29. Ladrón de Guevar C, Gonzales J, Peña P. Bacteraemia caused by Campylobacter spp. J Clin Pathol 1994:47:174-5.

30. Spelman DW, Davidson N, Buckmaster ND. Spicer WJ, Ryan P. Campylobacter bacteraemia: a report of 10 cases. Med J Austr 1986;145:503-5.

31. Meyrieux V, Monneret G, Lepape A, Chomarat M, Banssillon V. Fatal septic shock with multiple organ failure due to Campylobacter jejuni. Clin Infect Dis 1996:22:183-4.

32. Reed R, Friedland I, Wegerhoff FO. Khoosal M. Campylobacter bacteremia in children. Pediat Infect Dis J 1996;15: 345-8.

33. Schuster M. Blaser MJ. Nachamkin I. Serendipitous detection of persistent Campylobacter jejuni subspecies jejuni bacteremia in a patient undergoing bone marrow transplantation. Clin Infect Dis 1997;24:1270.

34. Mejer PA. Dooley DP, Jorgensen JH, Sanders C. Huang WM, Patterson JE. Development of quinolone-resistant Campylobacter fetus bacteremia in Human Immunodeficiency Virus-infected patients. J Infect Dis 1998:177:951-4.

35. Manfredi R. Nanetti A, Ferri M, Chiodo F. Fatal Campylobacter jejuni bacteraemia in patients with AIDS. J Med Microbiol 1999;48:601-3.

36. Ruiz-Contreras J, Ramos JT, Hernández-Sampelayo T, de José M, Clemente J, Gurbindo MD. Campylobacter sepsis in Human Immunodeficiency Virus-infected children. Pediat Infect Dis J 1997:16:251-3.

37. Allos BM, 'Lippy FT. Carlsen A, Washburn RG. Blaser MJ. Campylobacter jejuni strains from patients with Guillain-Barré syndrome. Emerg Infect Dis 1998;4:263-8. 
38. Camahan AM. Beadling J, Watsky D, Ford N. Detection of Campylubacter upsaliensis from a blood culture by using the BacT/Alert System. J Clin Microbiol 1994;32:2598-9.

39. Wilson ML. Mirrett S. Recovery of select rare and fastidious microorganisms from blood cultures. Clin Lab Med 1994:14: $119-31$.

40. Wang WLL. Blaser MJ. Detection of pathogenic Campylobacter specics in blood culture systems. J Clin Microbiol 1986:709-14. 41. Bastard JP, Le Pennec MP, Giacomini T. L'isolement par hémocultures des Campylobacter n'est plus une rareté. Presse Méd 1990;19:431.

42. Reimer LG, Wilson ML, Weinstein MP. Update on detection of bacteremia and fungemia. Clin Microbiol Rev 1997;10:444-65.

43. Frost JA, Kramer JM, Gillanders SA. Phage typing of Campylobacter jejuni and Campylobacter coli and its use as an adjunct to serotyping. Epidemiol Infecl 1999;123:47-55.

44. Smith KE, Besser JM. Hedberg CW, Leano FT, Bender JB. Wicklund JH, Johnson BP, Moore KA, Osterholm MT. Quinoloneresistant Campylobacter jejuni infections in Minnesota, 1992-1998. Investigation Team. N Engl J Med 1999:340:1525-32.

45. Maccario M. Tarantino A, Nobile-Orazio E, Ponticelli C. Campylobacter jejuni bacteremia and Guillain-Barré syndrome in a renal transplant recipienț. Transpl Int 1998:11:439-42.
46. Nachamkin I, Allos BM, Ho T. Campylobacter species and Guillain-Barré-syndrome. Clin Microbiol Rev 1998;11:555-6.

47. Blaser MJ. Editorial Response: Campylobacter fetus-Emerging infection and model system for bacterial pathogenesis at mucosal surfaces. Clin Infect Dis 1998;27:256-8

48. Ein Ausbruch von Campylobacter-Enteritis in Nordrhein-Westfalen. Epid Bull 1999:43:317-20.

49. Sakran W, Raz R, Levi Y, Colodner R, Koren A. Campylobacter bacteremia and pneumonia in two splenectomized patients. Eur J Clin Microbiol Infect Dis 1999:18:496-8.

50. Blaser MJ. Campylobacter and related species. In Mandell GL, Bennett JE, Dolin R (editors). Principles and Practice of Infectious Diseases. Churchill Livingstone, New York, N.Y. 1995:1948-56.

51. Nachamkin I. Campylobacter and Arcobacter. In: Murray PR, Baron EJ, Pfaller MA. Tenover FC, Yolken RH (editors), Manual of Clinical Microbiology. ASM Press Washington, D. C. 1999:716-26. 52. Pulik M. Genet P, Leturdu F, Lionnet F. Louvel D. Touahri T. Rifabutin prophylaxis against Mycobacterium avium complex infections in HIV-infected patients: impact on the incidence of campylobacteriosis. AIDS Patient Care STDS 1999;13(8):467-72.

53. Peetermans WE, De Man F, Moerman P. Van de Werf F. Fatal prosthetic valve endocarditis due to Campylobacter fetus. J Infect 2000;41(2):180-2. 